\title{
Desenvolvimento da estimativa numérica de quantidades no ensino fundamental
}

\section{Development of numerical estimate in primary education}

\author{
Mariana Lima Duro ${ }^{1}$ \\ Beatriz Vargas Dorneles ${ }^{2}$
}

\begin{abstract}
Resumo: Há um conjunto de evidências que tem relacionado a habilidade de realizar estimativa numérica ao desempenho matemático. Entretanto, observa-se que os resultados trazidos por estudos desta área são ainda inconsistentes e, muitas vezes, contraditórios. Nesta perspectiva, este estudo tem como objetivo caracterizar o desenvolvimento da estimativa numérica de quantidades discretas em estudantes do 20 ao 60 ano escolar. Foi realizado um estudo transversal quantitativo, com objetivo de observar possíveis níveis de desenvolvimento da habilidade de realizar estimativas. Os resultados foram obtidos a partir do cálculo da precisāo relativa, a partir de um Teste de Estimativa Numérica de Quantidades (TENQ), e indicaram diferentes níveis de desenvolvimento para cada situaçāo apresentada nas tarefas. Concluiu-se que a estimativa numérica é desenvolvida em crianças, passando por diferentes níveis de precisāo. A partir disso, sugere-se que a estimativa numérica de quantidades seja uma habilidade que pode ser desenvolvida e aprimorada ao longo de toda a vida.
\end{abstract}

Fecha de recepción: 27 de marzo de 2020. Fecha de aceptación: 04 de agosto de 2020.

1 Instituto Federal de Educaçāo, Ciência e Tecnologia do Rio Grande do Sul (IFRS), mariana.duro@ canoas.ifrs.edu.br, orcid ${ }^{\circ} \mathrm{rg} / 0000-0001-5492-4098$

2 Universidade Federal do Rio Grande do Sul (UFRGS), Faculdade de Educaçāo, beatriz.dorneles@ufrgs. br, orcid ${ }^{\circ} r g / 0000-0002-0141-9140$ 
Palavras-chave: Estimativa numérica de quantidades, desenvolvimento matemático, precisāo, matemática no ensino fundamental, numerosidade

\begin{abstract}
There is evidence that has related the ability of numerical estimation to mathematical performance. However, it is observed that the results brought by studies in this area are still inconsistent and, often, contradictory. Thus, the present study aims to understand the development of number Estimation of Discrete Quantities (ENQ), in students from the 2nd to the 6th school. A quantitative cross-sectional study was carried out to observe possible levels of development of the ability to make estimations. The results were obtained from the calculation of the relative precision, based on a Number Estimation of Quantities Test (TENQ), and indicated different levels of development for each situation presented in the tasks. It was concluded that the number estimate is developed in children, through different levels of accuracy. Due to this result, it was suggested that the number estimation of quantities to be a skill that can be developed and improved throughout life.
\end{abstract}

Keywords: Numerical estimation of quantities, mathematical development, accuracy, mathematics in elementary school, numerical estimate

\title{
1. INTRODUÇÃO
}

A competência matemática em geral compreende uma grande variedade de diferentes habilidades e processos cognitivos (Schneider, Grabner e Paetsch, 2009) e é importante para uma vida bem-sucedida na sociedade (Cirino, Tolar, Fuchs e Huston-Warren, 2016). Nas discussōes sobre quais habilidades matemáticas influenciam a obtenção de sucesso na sociedade adulta, encontra-se a estimativa numérica (Siegler e Braithwaite, 2016), responsável, justamente, por representar quantidades de forma rápida e sem (ou com pouca) necessidade de precisão.

Entretanto, embora as habilidades necessárias para a compreensão da estimativa numérica sejam muito básicas e enfatizadas na escola já nos primeiros anos escolares, a própria estimativa raramente tem sido discutida em sala de aula desde muito tempo. Ainda, nos documentos oficiais que orientam o trabaIho docente no Brasil, a estimativa está associada a quantidades ou ao resultado de uma conta (Plicastro, Rodrigues e Ribeiro, 2017). Como reflexo disso, a 
conclusāo mais consistente de investigaçōes sobre o desenvolvimento da estimativa é que as crianças não realizam estimativas com muita habilidade (Siegler e Booth, 2004), mesmo que no $5^{\circ}$ ano escolar já tenham adquirido habilidades suficientes (e até habilidades superiores) para compreender estimativas (Hurley, Boykin e Allen, 2005).

A grande variedade de tarefas que podem envolver estimativas exige que este estudo se concentre apenas em um campo da estimativa, sendo escolhida a Estimativa Numérica de Quantidades discretas em um conjunto (ENQ). Sendo assim, define-se ENQ como o número cardinal que representa a quantidade aproximada de elementos de um conjunto de objetos, sem que seja feita a contagem. Então, realizar estimativas de quantidades envolve atribuir um número a um conjunto de elementos discretos. Essa decisão reflete o interesse em compreender o desenvolvimento deste processo, em crianças do $2^{\circ}$ ao $6^{\circ}$ ano escolar, de modo a reduzir os conhecimentos externos exigidos para a realização das tarefas, como o conhecimento de unidades de medida ou conhecimentos específicos sobre informaçōes do mundo real.

Os pesquisadores Silva, Farias e Lima (2020) realizaram um estado da arte sobre a temática da estimativa numérica no Brasil, de 2000 a 2019. Como esperado, apenas onze artigos foram encontrados (sendo quatro de autoria de pelo menos uma das autoras deste trabalho) e categorizados em: reta numérica; áreas e medidas; quantitativo; aritmética e teórico. No caso da categoria "quantitativo", apenas 3 artigos encontrados, sendo um deles das autoras deste. Esta é mais uma evidência de que a estimativa numérica, em todos os seus desdobramentos, ainda é muito pouco discutida no Brasil e, quando se trata de estimativa numérica de quantidades, esta falta de estudos estende-se para o âmbito internacional.

Neste contexto, nesse estudo pretendeu-se responder às seguintes perguntas: a) Como se dá o desenvolvimento da ENQ em crianças do $2^{\circ}$ ao $6^{\circ}$ ano escolar nas diferentes situaçōes de apresentação dos estímulos? b) seria este desenvolvimento progressivo de modo que envolva níveis de desenvolvimento semelhantes para a mesma etapa escolar? c) de que maneira o modo de apresentação (diferentes escalas, diferentes densidades, com ou sem matriz de referência) das quantidades pode influenciar na precisão das estimativas nos diferentes anos escolares? Em síntese, o objetivo deste estudo foi comparar o desempenho das crianças em diferentes tarefas de enumeração das quantidades.

Esta pesquisa integra um projeto mais abrangente, intitulado "Diversidade na aprendizagem da matemática inicial: a compreensão da estimativa numérica" (Plataforma Brasil e Comitê de Ética em Pesquisa da Universidade Federal do 
Rio Grande do Sul, sob o número 31575913.6.0000.5347). O projeto tem como proposta o estudo da diversidade na aprendizagem da matemática, no que se refere à compreensão da estimativa numérica em diferentes grupos de alunos.

\section{REFERENCIAL TEÓRICO}

Estimativa numérica estaria relacionada à capacidade numérica das pessoas, que, por sua vez, está relacionada ao Senso Numérico (SN). Entretanto, não há consenso sobre a origem do SN. A neuropsicologia indica que a localização neuronal do SN é a mesma da quantificação exata (Dehaene, 1997), sendo o SN uma capacidade inata que possibilita que algumas pessoas nasçam com maior capacidade numérica que outras. Essas ideias vão de encontro à perspectiva construtivista de que o SN é construído e progressivamente organizado internamente e que possibilita a interação com números e o desenvolvimento de estratégias eficientes para esta interação (Corso e Dorneles, 2012).

Considera-se que a habilidade de realizar ENQ vai além do SN, no sentido que Dehaene (1997) propōe, e pode se desenvolver com a idade e com a experiência educacional e cultural, permitindo o avanço desta habilidade conforme o desenvolvimento do sistema simbólico da criança (Halberda e Feigenson, 2008). Corroborando este entendimento, Leibovich, Katzin, Harel e Henik (2017) colocam em cheque as teorias de que o senso numérico é inato e a teoria que indica que a representação de quantidades não simbólicas está sendo processada independentemente de magnitudes contínuas (por exemplo, tamanho, área e densidade), concluindo que não há evidências suficientes para sustentar a teoria do senso numérico inato. Para dar conta desse entendimento, os autores (Leibovich et al., 2017) sugerem um "senso de magnitude" desenvolvido a partir da compreensāo da correlaçāo entre numerosidade e magnitudes contínuas.

Alguns pesquisadores (Opfer e Siegler, 2012) indicam que o pensamento quantitativo não-simbólico está presente desde muito cedo na criança, sendo ele culturalmente universal e semelhante entre as espécies, em especial, na capacidade de representar e comparar quantidades. Essa forma de representação mental nāo-verbal primitiva, que suporta um senso numérico intuitivo e inato em humanos e outras espécies de animais, é chamada de Sistema de Número Aproximado (ANS: Aproximate Number System).

Anterior à quantificação exata mais elaborada, alguns pesquisadores afirmam que a enumeração de objetos brevemente apresentados é precisa para 
até 3 ou 4 itens e que o reconhecimento de quantidades entre "um" e "quatro" parece ser anterior à aquisição dos princípios de contagem (Dehaene, 1997). Essa capacidade, presente em diferentes espécies, de identificar precisa e rapidamente pequenas quantidades numéricas é chamada de "subitizing". Embora o subitizing tenha sido estudado extensamente desde muito tempo, seus mecanismos subjacentes permanecem sendo debatidos.

Ainda que Feigenson et al. (2004) tenham ampliado o conceito original de senso numérico, propondo a existência de dois sistemas de representação numérica distintos em animais humanos e não humanos (um para quantidades menores ou igual a quatro e outro para quantidades maiores), os autores afirmam que o sistema que permite representação de numerosidades não simbólicas é usado posteriormente para um pensamento matemático complexo. Entretanto, ao comparar dois conjuntos de estímulos de diferentes numerosidades, além da quantidade, existem outras diferenças, tais como o comprimento total do contorno e a área total da superfície (Leibovich, Katzin, Harel e Henik, 2017).

Apesar da concordância geral entre os pesquisadores de que a enumeraçāo é tratada de forma diferente dentro do intervalo de subitizing, tem havido algum debate sobre se o subitizing utilizaria mecanismos iguais ou diferentes daqueles utilizados na estimativa de intervalos numéricos maiores. Entretanto, deve-se ter o devido cuidado para não tratar as ENQ como habilidades integralmente perceptivas, tal como se considera ser o caso do subitizing. Por esse motivo, não se incluiu nesta pesquisa o conceito de subitizing, já que parece envolver um processo diferente do que o envolvido em estimativas de conjuntos maiores.

Em vez disso, Gebuis e Reynvoet (2012) propōem que os seres humanos estimam quantidades através da ponderação dos diferentes elementos visuais presentes nos estímulos. Por exemplo, quando maçãs são adicionadas a uma pilha de maçãs, o tamanho da pilha aumenta; ou quando mais pessoas entram em uma sala, a densidade aumenta. Portanto, a informação visual é importante para a estimativa de quantidades, mas não é suficiente para estabelecer estimativas precisas, o que exigiria muito mais que padrōes perceptivos ou sensibilidades inatas de quantificação.

Para realizar ENQ, tal como sugerido neste estudo, requer-se um mecanismo logicamente mais elaborado. Ou seja, para fazer apenas julgamentos de menor/ maior, considera-se que estas pistas visuais possam ser, de alguma forma, suficientes, entretanto, para ENQ mais precisas, elas não seriam suficientes. Dessa forma, o ANS não é um mecanismo relevante para este estudo. 
Alguns autores (Dehaene, 1997) trabalham com a ideia de que a discriminação de quantidades seria uma atividade ligada apenas à percepção, propondo atividades que envolvem somente relacionar duas quantidades, escolhendo qual grupo apresenta mais/menos elementos. Corroborando esta ideia, Rousselle e Noel (2008) constataram que o desempenho em uma tarefa de ENQ é afetado por propriedades numéricas e perceptivas. Os pesquisadores concluíram que a automatização do processamento de quantidades surge gradualmente ao longo do desenvolvimento, ao passo que o acesso automático à informação perceptiva já está bem desenvolvido em pré-escolares. Ou seja, se a estimativa de quantidades fosse uma atividade integralmente perceptiva, as crianças deveriam ser capazes de fazê-la desde muito cedo, o que nāo ocorre.

Em contrapartida, Nogues e Dorneles (2020) afirmam que o raciocínio quantitativo está envolvido nas relaçōes entre as quantidades, sendo fundamental para a construção dos conceitos primitivos da matemática. As autoras apresentam os conceitos de Nunes e colaboradores (2016) para explicar o raciocínio aditivo (parte-todo) e multiplicativo (correspondência um para muitos), enquanto relaçōes entre quantidades. Concorda-se com as autoras, entendendo que representar numericamente quantidades também pode envolver essas relaçōes, já que as estratégias de estimativa, à medida que se tornam mais complexas e relacionais, também se tornam mais precisas.

Nessa perspectiva, Kim, Jang e Cho (2018) realizaram um estudo com os objetivos de testar se (e quais) habilidades numéricas básicas podem ser desenvolvidas a partir de treinamento e se os efeitos do treinamento são transferidos para melhorias no desempenho geral da matemática. Os autores indicam que a literatura traz evidências contraditórias sobre a eficácia do treinamento da habilidade numérica básica. Como resultado, não foram encontradas evidências de que os efeitos do treinamento ocasionaram melhoria em qualquer domínio do desempenho matemático, embora perceberam melhora na precisāo da comparaçāo de quantidades (ANS). Entretanto, embora os autores nāo tenham encontrado relação direta para este treinamento, não se pode descartar a possibilidade de que essa aprendizagem tenha se dado em outros domínios ou componentes de habilidades matemáticas não testadas.

Em estudo preliminar de Dorneles e colaboradores (2015), em que estudantes foram solicitados a realizar estimativas acerca da quantidade de círculos em uma imagem, comparando conjuntos, observou-se que, quando os grupos de círculos são iguais (ou muito semelhantes) em quantidade, a categoria com o mais elevado grau de aglomeração (ou repetição) é julgada mais numerosa pela 
criança. Esses resultados foram ao encontro dos obtidos por Piazza e colaboradores (2006), que observaram que quando os grupos de itens são iguais em número, o grupo de maior aglomeração é julgado mais numeroso. Contribuindo com estes achados, Brysbaert (2005) já havia concluído que a enumeração das quantidades maiores é mais fácil quando os itens são apresentados em uma forma canônica do que quando são apresentados em uma configuração aleatória.

O estudo de Kersey, Braham, Csumitta, Libertus e Cantlon (2018) examinou diferenças de gênero na cognição matemática por meio de teste que avaliou a percepção de quantidades, a contagem e os conceitos formais e informais de matemática elementar. Como o esperado, não foram observadas diferenças por gênero. Entretanto, os autores indicam que, às vezes, diferenças podem depender de geração ou escola. Essa suposição é corroborada no estudo de Siegler, e Braithwaite (2016), no qual os autores afirmam que o conhecimento numérico de crianças em idade pré-escolar de baixa renda fica, em média, pelo menos um ano atrás do dos seus pares de famílias de renda média e que essas diferenças iniciais também têm consequências a longo prazo. Ainda nesta perspectiva, o estudo de Cirino, Tolar, Fuchs e Huston-Warren (2016) indicou que o desempenho em estimativa numérica de quantidades está relacionado a uma grande variedade de preditores, sendo a idade e etnia foram significativamente relacionadas a essa capacidade, ao contrário do sexo.

Sugere-se que os resultados dos testes de ENQ podem ser mais confiáveis que o de outras tarefas de estimativa numérica pela familiaridade das crianças brasileiras com tarefas de quantificação, mesmo que estas sejam com foco nas quantificaçōes exatas de itens. Ou seja, a partir de relatos de professores que ensinam os fundamentos da aritmética para crianças, percebe-se ser muito comum realizar atividades de representação numérica a partir da comparação do símbolo numérico a sua quantidade, utilizando como suporte objetos como pedrinhas, balas ou outros itens familiares às crianças.

Uma tarefa utilizada para testar a ENQ, a qual também será utilizada neste estudo, configura-se em observar itens dispostos em matrizes quadradas por um determinado período de tempo, de modo que não seja possível contá-los, mas que seja suficiente para o estabelecimento de estratégias que possibilitem uma estimativa mais precisa que a obtida apenas por percepção visual dos itens. Ou seja, essa tarefa nada tem a ver com simplesmente comparar conjuntos, indicando qual possui mais ou menos itens. 


\section{MÉTODO}

Foi realizado um estudo transversal, com uma amostra de 676 estudantes do $2^{\circ}$ $(n=116), 3^{\circ}(n=127), 4^{\circ}(n=94), 5^{\circ}(n=163)$ e $6^{\circ}(n=176)$ anos escolares. Estes estudantes formavam a população de duas escolas: uma escola pública municipal ( $\mathrm{n}=389)$ e uma escola privada ( $\mathrm{n}=287)$, ambas na cidade de Porto Alegre/ RS - Brasil. A frequência da amostra por ano escolar, escola e gênero estão descritos na Tabela 1. Os alunos pesquisados aceitaram participar da pesquisa e foram liberados de suas atividades de sala de aula pelo professor, apresentando autorização dos pais ou responsáveis para participar do projeto.

Como não há relatos de um teste que avalie o desempenho em estimativa numérica de quantidades de acordo com a precisão relativa (PR) individual e considerando as mesmas variáveis deste estudo, o Teste de Estimativa Numérica de Quantidades (TENQ) foi estruturado e organizado pelas pesquisadoras deste estudo. O TENQ avalia a habilidade do sujeito de realizar estimativas numéricas da quantidade de objetos em um conjunto discreto, considerando o tempo destinado para tal, de forma a impedir a contagem os itens.

O instrumento é composto por 64 tarefas, subdivididas em diferentes formas de apresentaçāo e configuraçōes espaciais de seus estímulos, que consistiram em conjuntos de pontos de diferentes quantidades, de mesma cor (preta) e de mesmo tamanho (a fim de reduzir a quantidade de variáveis que possam interferir no julgamento da quantidade pelos participantes). Eles foram irregularmente posicionados sobre a regiāo delimitada pela tela retangular, projetada em um fundo branco. Estímulos não simbólicos são úteis no estudo do processamento de numerosidades, já que eles podem ser usados com diferentes populaçōes adultos, bebês e animais (Leibovich, Katzin, Harel e Henik, 2017). Entretanto, estímulos nāo simbólicos também contém contornos contínuos de magnitudes nāo numéricas.

Para impedir os participantes de usar magnitudes contínuas para fazer sua decisão, foram propostas diferentes formas de apresentação dos estímulos, de modo que é praticamente impossível obter dois grupos de itens que variam apenas em termos de numerosidade (Leibovich, Katzin, Harel e Henik, 2017). O intervalo de quantidades foi estendido até 100, maior do que a maioria dos estudos anteriores, que incluíram quantidades até 80 (Kim, Jang e Cho, 2018). Além disso, variou-se as informaçōes não numéricas, a partir de diferentes propriedades visuais dos estímulos não simbólicos (pontos e matrizes), para que outras influências fossem minimizadas. 
Para atingir os objetivos a que se propõe, observou-se a necessidade de contemplar diferentes intervalos numéricos (escalas), representados pelas diferentes matrizes $(10 \times 1=E 10,10 \times 2=E 20$ e 10x10=E100), pressupondo diferentes níveis de dificuldade, considerando-se que a habilidade de realizar estimativas é processual e ocorre de forma gradual. Há indícios de que as estratégias e a precisão na realização de estimativa variam com o formato de apresentação (aglomerados (A) - itens apresentados lado a lado na matriz -, espaçados (E) - itens aleatoriamente distribuídos na matriz - e aleatórios (AL) - sem auxílio da matriz) e com o fato de conhecer ou desconhecer o número máximo de pontos em cada matriz (intervalo de valores possíveis). Todas as variáveis interagiram entre si.

O procedimento para a realização do TENQ consistiu em avaliar os estudantes separados por turma, dentro da sala de aula, de forma coletiva, de maneira que o ambiente de trabalho e suas atividades fossem o menos afetado possível. O tempo médio de realização do teste foi de 50 minutos por turma. As tarefas do TENQ foram apresentadas visualmente, com auxílio do software PowerPoint e de um projetor multimídia.

A tarefa consistia em ouvir a instrução, observar a imagem projetada e, após alguns segundos de observação, a imagem era retirada da tela, seguida por uma tela branca. Neste momento, os participantes eram convidados a realizar uma estimativa numérica acerca da quantidade observada, anotando em um caderno de respostas previamente distribuído a quantificação que considerassem mais adequada para a imagem apresentada. Para cada dezena de itens, um segundo a mais era dado para observação. Ou seja, para quantidades de até 10 elementos, os participantes tinham um segundo de observação; para quantidades de 11 a 20, eram disponibilizados 2 segundos, de 21 a 30, 3 segundos e, assim por diante, até o período máximo de 10 segundos, para quantidades entre 91 e 100 elementos. Não houve feedback quanto às respostas na intenção de evitar um aprendizado perceptual.

Para compreender o desenvolvimento da estimativa numérica de quantidades, foi realizada uma análise estatística, considerando a variável dependente precisão relativa (PR) em cada uma das escalas (E10, E20 e E100), em cada ano escolar $\left(2^{\circ}, 3^{\circ}, 4^{\circ}, 5^{\circ}, 6^{\circ}\right)$, nas diferentes escolas (pública-Pu ou privada-Pa). Ainda dentro de cada escala, as variáveis consistiam em a criança conhecer o máximo de pontos possíveis em cada matriz ou não [máximo conhecido (MC) / máximo desconhecido (MD)] e os pontos estarem distribuídos de forma aglomerada (A), espaçadas (E) na matriz ou aleatória (AL), sem matriz auxiliar. 
Considerando que a precisão das estimativas possa fornecer, de forma confiável, informação sobre como os indivíduos realizam juízos de enumeração, em primeiro lugar, para cada problema e para cada aluno, tanto a precisão absoluta (PA) quanto a relativa (PR) foram calculadas. Para o cálculo da PR, utilizou-se a fórmula adaptada de Siegler e Booth (2004), sendo o Valor Real o número a ser estimado e Estimativa o valor determinado pelo sujeito, então: $P R=\frac{\mid \text { VALOR REAL-ESTIMATVAI }}{E S C A L A(10,200 \mathrm{ou} 100)}$. Dessa forma, quanto mais próximo de zero for o resultado da PR, mais precisa é esta estimativa. O resultado obtido com o cálculo realizado no numerador da fração é o que se chama de precisão absoluta (PA). Neste caso, $p a=\mid$ valor real - estimativa $\mid$.

Um nível de confiança alfa de 0,05 foi utilizado para todos os testes estatísticos. Os p-valores exatos foram relatados, mas valores muito pequenos foram arredondados para $p<0,001$. A partir do teste de normalidade de Shapiro-Wilk, foi verificada a distribuição da variável precisão relativa (PR). Apresentando distribuição assimétrica, optou-se por utilizar o teste não paramétrico de Mann-Whitney para comparar a distribuição da PR em relação às categorias de sexo ( $\mathrm{M}$ e F) e escola (pública e particular). Não houve diferença significativa para sexo em nenhuma das escalas, entretanto, o mesmo não ocorreu para as diferentes escolas. Por isso e pelos indícios apontados pela literatura, optou-se por realizar as análises separando os resultados também por escola.

Para a análise estatística da relação entre as variáveis, utilizou-se a Análise de Modelo de Equaçōes de Estimaçōes Generalizadas (GEE), tendo em vista que o mesmo sujeito respondeu a várias questōes de um mesmo teste. Também se utilizou uma matriz de correlação trabalho Exchangeable e uma matriz de covariância de estimador robusto, considerando-se estes dois testes mais utilizados na literatura para variáveis assimétricas e para uma distribuição normal com função identidade.

Optou-se pela utilização da distribuição normal (mesmo a variável não sendo simétrica) e não a distribuição gama (logarítmica), pois os casos em que o indivíduo acerta o valor real (ou seja, que sua precisão é zero) seriam excluídos, já que o logaritmo de zero não está definido. O teste post-hoc utilizado foi o teste de comparação múltipla de Bonferroni. 


\section{RESULTADOS}

Realizou-se um modelo fatorial entre as quatro variáveis: máximo conhecido ou desconhecido (MC/MD); aglomerado, espaçado ou aleatório (A/E/AL); ano (2o$60)$ e escola pública ou escola particular ( $\mathrm{Pu} / \mathrm{Pa}$ ), discriminando suas relaçōes em cada uma das três diferentes escalas (E10, E20 e E100). Para realizar esta primeira análise, fixou-se as variáveis ano e escola e comparou-se as médias dos estudantes em cada uma das escalas. Tanto para a escola pública quanto para a particular, a interação entre as variáveis escala e ano foi significativa ( $p<0,001$ e $p=0,001$, respectivamente) e a média geral de PR por escala na escola pública é: $E 10=0,0557 ; E 20=0,0690 ; E 100=0,0904 ; A L=0,1046$ e na escola particular é: $\mathrm{E} 10=0,0389 ; \mathrm{E} 20=0,0470 ; \mathrm{E} 100=0,0618 ; \mathrm{AL}=0,0933$, de acordo com as diferenças culturais já apontadas por Cirino e Colaboradores (2016), Kersey e colaboradores (2018) e Siegler e Braithwaite (2016).

Esses dados sugerem que estimativas em escalas menores são mais precisas que em escalas maiores e determinam uma necessidade de analisar as diferentes tarefas não apenas por ano escolar (como é objetivo do estudo), mas também por escola e a partir de cada uma das escalas. Sendo assim, em um primeiro momento, realizou-se a comparação das médias da PR por ano escolar e por escola, nas diferentes escalas. Esses dados foram detalhados na Tabela 1.

Tabela 1. Médias da Precisāo Relativa por ano e escola nas diferentes escalas

\begin{tabular}{|c|c|c|c|c|c|c|c|c|}
\hline \multirow{3}{*}{ Ano } & \multicolumn{8}{|c|}{ Escola } \\
\hline & \multicolumn{4}{|c|}{ Pública } & \multicolumn{4}{|c|}{ Particular } \\
\hline & E10 & E20 & E100 & $\mathrm{AL}$ & E10 & E20 & E100 & $\mathrm{AL}$ \\
\hline $2^{\circ}$ & 0,0867 & 0,124 & 0,1419 & 0,1441 & 0,0588 & 0,0626 & 0,0768 & 0,1211 \\
\hline $3^{\circ}$ & 0,0943 & 0,0949 & 0,1177 & 0,1441 & 0,049 & 0,0463 & 0,0687 & 0,0787 \\
\hline $4^{\circ}$ & 0,0796 & 0,0852 & 0,1166 & 0,1177 & 0,0447 & 0,0485 & 0,0631 & 0,112 \\
\hline $5^{\circ}$ & 0,0418 & 0,0509 & 0,0846 & 0,0864 & 0,0345 & 0,0411 & 0,0591 & 0,1024 \\
\hline $6^{\circ}$ & 0,0324 & 0,0347 & 0,0702 & 0,0916 & 0,0304 & 0,037 & 0,0634 & 0,0913 \\
\hline
\end{tabular}

Fonte: autoras 
Em uma análise vertical da Tabela 1, observa-se que, à medida que aumenta o ano escolar, as médias das precisōes tendem a diminuir, exceto para os anos escolares destacados. Ou seja, em geral, quanto maior o nível de escolaridade dos alunos, mais precisos eles são em suas estimativas. A média dos itens aleatórios apresentadas pelo $3^{\circ}$ ano na escola particular mostrou-se muito superior às demais dos anos posteriores, por isso destacada na Tabela 1. Talvez esse resultado esteja relacionado a etapa escolar desses estudantes, que prevê o estudo do número enquanto indicador de quantidade de elementos de uma coleção discreta (cardinalidade), também quantificando coleçōes ou eventos a partir da correspondência biunívoca e da conservação da quantidade pela relação entre: quantidade/quantidade quantidade/símbolo, símbolo/quantidade e agrupamentos.

É fácil ver também que as médias dos alunos da escola particular representam estimativas mais precisas que seus pares de escola pública (exceto no $5^{\circ}$ ano para itens aleatórios e no $6^{\circ}$ ano para E20). Das diferenças em termos de média de Precisão Relativa apontadas na Tabela 1, obtém-se as significativas, em cada escala, na Tabela 2.

A partir destes dados, pode-se observar que as possíveis mudanças em termos de desenvolvimento da estimativa ocorrem em diferentes etapas para cada escola. Observa-se que, no caso da escola pública, em geral, os três primeiros anos não apresentam diferenças estatísticas entre eles, seguido de um grupo intermediário, cujas médias de precisāo ora assemelham-se ao grupo de menor ano escolar, ora ao grupo de alunos de estágio escolar mais avançado. Entretanto, observa-se um padrão interessante, dado que a precisão dos estudantes do $2^{\circ}$ e do $3^{\circ}$ ano diferem estatisticamente das dos $5^{\circ}$ e do $6^{\circ}$ ano em todas as formas de distribuição. Diferentemente, na escola particular, apenas a precisão do $2^{\circ}$ ano diferiu estatisticamente da do $5^{\circ}$ e do $6^{\circ}$ ano e apenas nas duas escalas menores. Esses dados indicam que as crianças menores, da escola pública, têm suas estimativas piores que o mais velhos em todas as formas de apresentação dos estímulos, enquanto que, na escola particular essa diferença só ocorre para as escalas menores e, supostamente, mais fáceis de serem estimadas, indicando que as estimativas dos estudantes da escola particular são mais precisas desde os primeiros anos escolares. 
Tabela 2. Diferenças significativas entre as médias de Precisāo Relativa por ano, escola e escalas

\begin{tabular}{|c|c|c|c|c|c|c|c|}
\hline Escala & Ano & $3^{\circ}$ & $\mathrm{p}$ & $5^{\circ}$ & $p$ & $6^{\circ}$ & $p$ \\
\hline \multirow{4}{*}{ E10 } & \multirow{2}{*}{$2^{\circ}$} & & & $\mathrm{Pu}$ & 0,001 & $\mathrm{Pu}$ & $<0,001$ \\
\hline & & & & $\mathrm{Pa}$ & $<0,001$ & $\mathrm{~Pa}$ & $<0,001$ \\
\hline & $3^{\circ}$ & & & $\mathrm{Pu}$ & 0,005 & $\mathrm{Pu}$ & $<0,001$ \\
\hline & $4^{\circ}$ & & & & & $\mathrm{Pu}$ & 0,043 \\
\hline \multirow{5}{*}{ E20 } & \multirow{2}{*}{$2^{\circ}$} & & & $\mathrm{Pu}$ & $<0,001$ & $\mathrm{Pu}$ & $<0,001$ \\
\hline & & & & $\mathrm{Pa}$ & 0,013 & $\mathrm{~Pa}$ & $<0,001$ \\
\hline & $3^{\circ}$ & & & $\mathrm{Pu}$ & $<0,001$ & $\mathrm{Pu}$ & $<0,001$ \\
\hline & $4^{\circ}$ & & & & & $\mathrm{Pu}$ & 0,015 \\
\hline & $5^{\circ}$ & & & & & $\mathrm{Pu}$ & $<0,001$ \\
\hline \multirow{3}{*}{ E100 } & $2^{\circ}$ & & & $\mathrm{Pu}$ & $<0,001$ & $\mathrm{Pu}$ & $<0,001$ \\
\hline & $3^{\circ}$ & & & $\mathrm{Pu}$ & 0,017 & $\mathrm{Pu}$ & $<0,001$ \\
\hline & $4^{\circ}$ & & & $\mathrm{Pu}$ & 0,014 & $\mathrm{Pu}$ & $<0,001$ \\
\hline \multirow{3}{*}{$\mathrm{AL}$} & $2^{\circ}$ & $\mathrm{Pa}$ & 0,003 & $\mathrm{Pu}$ & $<0,001$ & $\mathrm{Pu}$ & $<0,001$ \\
\hline & $3^{\circ}$ & & & $\mathrm{Pu}$ & $<0,001$ & $\mathrm{Pu}$ & 0,004 \\
\hline & $4^{\circ}$ & & & & & & \\
\hline
\end{tabular}

p - Nível de confiança 
A fim de categorizar os dados apresentados nas Tabelas 1 e 2 e, assim, destacar os diferentes níveis de desenvolvimento de estimativa numérica de quantidades, foram verificadas as semelhanças em termos de precisão dentre os grupos de alunos dos diferentes níveis escolares. Com a substancial diferença apresentada entre os resultados obtidos pelos alunos da escola pública em comparação aos da escola particular, fez-se necessário distinguir diferentes grupos para cada escala apresentada. A partir desse agrupamento, verificou-se que é possível determinar diferentes níveis de desenvolvimento da estimativa numérica de quantidade nas diferentes escalas, entretanto, alguns grupos (anos escolares) podem ser categorizados em dois ou mais níveis, de acordo com as semelhanças e diferenças estatísticas encontradas, em termos de precisão. Chamar-se-ão estes grupos de Grupos de Transição (GT).

Os níveis foram assim delimitados a partir das diferenças estatísticas apresentadas na Tabela 2. Ou seja, o nível 1 (N1) é composto pelos anos escolares que não obtiveram diferenças estatísticas a partir do $2^{\circ}$ ano até o ano que apresenta diferenças quando comparado a este. O nível 2 (N2) é composto pelos anos escolares que diferem do nível 1, mas não entre si. Da mesma maneira, distinguem-se os níveis 3 e 4 (N3 e N4), quando houver, compostos pelos anos escolares que diferem dos do N1 e do N2, mas que são iguais entre si, e assim sucessivamente. Em todos os níveis encontrados, pode haver GT que pertençam a mais de um grupo, tendo em vista as diferenças e as semelhanças de precisão quando comparados aos demais anos que pertencem ao grupo. Para facilitar o reconhecimento dos grupos por ano escolar, optou-se por apresentá-los na Tabela 3, destacando os GT em grifo.

Tabela 3. Categorizaçāo dos anos escolares em Níveis por média de PR nas diferentes escolas e escalas

\begin{tabular}{|c|c|c|c|c|c|c|c|c|}
\hline \multirow{3}{*}{ Escala } & \multirow{3}{*}{$\begin{array}{c}\text { Nível } \\
\text { N1 }\end{array}$} & \multicolumn{7}{|c|}{ Escola } \\
\hline & & \multicolumn{3}{|c|}{ Pública } & \multicolumn{4}{|c|}{ Privada } \\
\hline & & $2^{\circ}$ & $3^{\circ}$ & $4^{\circ}$ & $2^{\circ}$ & $3^{\circ}$ & $4^{0}$ & \\
\hline \multirow[t]{3}{*}{ E10 } & N2 & $4^{\circ}$ & $5^{\circ}$ & & $3^{\circ}$ & $4^{0}$ & $5^{\circ}$ & $6^{\circ}$ \\
\hline & N3 & $5^{\circ}$ & $6^{\circ}$ & & & & & \\
\hline & N1 & $2^{\circ}$ & $3^{\circ}$ & $4^{0}$ & $2^{\circ}$ & $3^{\circ}$ & $4^{0}$ & \\
\hline \multirow[t]{2}{*}{ E20 } & N2 & $4^{\circ}$ & $5^{\circ}$ & & $3^{\circ}$ & $4^{\circ}$ & $5^{\circ}$ & $6^{\circ}$ \\
\hline & N3 & $6^{\circ}$ & & & & & & \\
\hline
\end{tabular}




\begin{tabular}{llllllllll}
\hline & $\mathrm{N} 1$ & $2^{\circ}$ & $3^{\circ}$ & $4^{\circ}$ & $2^{\circ}$ & $3^{\circ}$ & $4^{\circ}$ & $5^{\circ}$ & $6^{\circ}$ \\
& $\mathrm{N} 2$ & $5^{\circ}$ & $6^{\circ}$ & & & & & & \\
& $\mathrm{N} 3$ & & & & & & & & \\
\hline \multirow{3}{*}{$\mathrm{AL}$} & $\mathrm{N} 1$ & $2^{\circ}$ & $3^{\circ}$ & $4^{\circ}$ & $2^{\circ}$ & $4^{\circ}$ & $5^{\circ}$ & $6^{\circ}$ & \\
& $\mathrm{N} 2$ & $4^{\circ}$ & $5^{\circ}$ & $6^{\circ}$ & $3^{\circ}$ & $4^{\circ}$ & $5^{\circ}$ & $6^{\circ}$ & \\
& $\mathrm{N} 3$ & $5^{\circ}$ & $6^{\circ}$ & & & & & & \\
\hline
\end{tabular}

Pode-se observar que, na escola pública, na maioria dos casos, originaram-se 3 diferentes níveis, enquanto que, na escola particular, apenas 2, exceto pela escala 100, que reduziu a quantidade de grupos para ambas as escolas. 0 primeiro nível foi composto pelos três primeiros anos escolares que participaram da pesquisa, sendo que o $6^{\circ}$ ano integrou o último nível em todos os casos. As constataçōes também permitem pensar que a apresentação $A L$, aleatória, é igualmente imprecisa para todas as crianças. Apenas na escala 100 pode-se verificar forte distinção entre as médias e isso pode estar relacionado aos maiores conhecimentos de proporção e de relações aritméticas dos mais velhos.

De maneira geral, vislumbramos um nível inicial de precisāo, composto pelos estudantes do $2^{\circ}$ ao $4^{\circ}$ ano, um possível nível intermediário, basicamente composto por GTs e, um grupo de estudantes mais precisos, em geral, composto por estudantes do $5^{\circ}$ e do $6^{\circ}$ ano. Esses níveis apresentam-se bem variáveis, dado que a estimativa não é ensinada e muito depende das experiências extraescolares dos estudantes.

\section{Comparação entre as variáveis Máximo Conhecido (MC)/Máximo Desconhe- cido (MD) nos diferentes Anos e Escalas}

Fixando escala 10 (E10) e realizando a interação com MD/MC, teve-se que a interação MD/MC com ano é significativa para a escola pública ( $p=0,007$, média $\mathrm{MD}=0,0409$; média $\mathrm{MC}=0,0359)$, mas não para a escola particular ( $\mathrm{p}=0,110$, média $M D=0,0522$; média $M C=0,0589$ ). Em E20, a interação MD/MC e ano não é significativa nem para a escola pública ( $p=0,615$; média $M D=0,0706$; média $M C=0,0674$ ), nem para a escola particular ( $\mathrm{p}=0,360$; média $M D=0,0539$; média $M C=0,0398$ ). Quando analisada a E100, obteve-se significativa a interação MD/MC com ano ( $p<0,001$ ), para ambas as escolas: pública (média $M D=0,1033$; média $M C=0,0767$ ) e particular (média $\mathrm{MD}=0,0790$; média $\mathrm{MC}=0,0448$ ). Consideraram-se apenas as 
médias apresentadas nos diferentes anos para quando o máximo é conhecido ou não, no caso da escala 100, tendo em vista ser essa interação significativa em ambas as escolas. Esses dados são apresentados na Tabela 4.

Tabela 4. Médias de PR para MD e MC por escola e ano escolar na Escala 100

\begin{tabular}{cccccc}
\hline \multirow{2}{*}{ Ano } & \multicolumn{2}{c}{ Escola Pública } & & \multicolumn{2}{c}{ Escola Privada } \\
\cline { 2 - 3 } \cline { 6 - 6 } & MD & MC & & MD & MC \\
\hline $2^{\circ}$ & 0,1465 & 0,1369 & & 0,0936 & 0,0603 \\
$3^{\circ}$ & 0,1172 & 0,1181 & & 0,0851 & 0,0523 \\
$4^{\circ}$ & 0,1406 & 0,088 & & 0,0888 & 0,0374 \\
$5^{\circ}$ & 0,1167 & 0,0519 & & 0,0815 & 0,0371 \\
$6^{\circ}$ & 0,0896 & 0,0511 & & 0,0912 & 0,036 \\
\hline
\end{tabular}

Neste caso, mais uma vez, foram encontradas diferenças substanciais entre a precisão das escolas pública e particular, em se tratando de desconhecer o máximo de pontos da matriz apresentada. Na escola particular, obtiveram-se médias não diferentes em todos os anos. Muito diferente ocorre na escola pública, na qual o máximo de pontos desconhecido influencia muito a precisāo dos estudantes por ano escolar. Neste caso, $2^{\circ}, 3^{\circ}$ e $4^{\circ}$ anos têm médias não diferentes, enquanto que $5^{\circ}$ e $6^{\circ}$ anos têm médias diferentes, sendo que o $6^{\circ}$ ano é diferente de todos os anos.

Pode-se dizer que conhecer o máximo de pontos da matriz influencia na precisão dos alunos dos anos escolares, nas diferentes escolas. Tanto para a escola pública quanto para a particular, os resultados foram bastante semelhantes, sendo $2^{\circ}$ e $3^{\circ}$ ano com médias não diferentes e $4^{\circ}, 5^{\circ}$ e $6^{\circ}$ anos também. 0 fato de os estudantes serem mais precisos ao saber o total de espaços disponíveis na matriz, indica que outras habilidades matemáticas estão envolvidas em suas estimativas, e que relaçōes aritméticas podem ter sido envolvidas nessa ação. Na Tabela 5 foi realizada a categorização por grupos, de acordo com a precisão apresentada pelos estudantes. 
Tabela 5. Categorizaçāo dos anos escolares em Níveis por PR para MC ou MD em cada escola

\begin{tabular}{|c|c|c|c|c|c|c|c|c|c|}
\hline \multirow{3}{*}{ MD/MC } & \multirow{2}{*}{ Nível } & & & & \multicolumn{5}{|c|}{ Escola } \\
\hline & & \multicolumn{3}{|c|}{ Pública } & \multicolumn{5}{|c|}{ Privada } \\
\hline & N1 & $2^{\circ}$ & $3^{\circ}$ & $4^{\circ}$ & $2^{\circ}$ & $3^{\circ}$ & $4^{\circ}$ & $5^{\circ}$ & $6^{\circ}$ \\
\hline \multirow[t]{3}{*}{ MD } & N2 & $3^{\circ}$ & $4^{\circ}$ & $5^{\circ}$ & & & & & \\
\hline & N3 & $6^{\circ}$ & & & & & & & \\
\hline & N1 & $2^{\circ}$ & $3^{\circ}$ & & $2^{\circ}$ & $3^{\circ}$ & & & \\
\hline \multirow[t]{2}{*}{$M C$} & N2 & $3^{\circ}$ & $4^{\circ}$ & & $4^{0}$ & $5^{\circ}$ & $6^{\circ}$ & & \\
\hline & N3 & $5^{\circ}$ & $6^{\circ}$ & & & & & & \\
\hline
\end{tabular}

Grifados, na Tabela 5, encontram-se destacados os Grupos de Transiçāo. Observa-se que os grupos constituídos a partir da variável MC/MD são bastante distintos, em especial na escola privada. Embora não obtendo padrōes mais precisos de agrupamento, os dados da Tabela 5 mostram que, também neste caso, os alunos com maior escolarização são mais precisos, indicando uma possível construção da habilidade de realizar estimativas de quantidades, mesmo quando não há mais informações sobre a matriz de referência, mas que esta informação pode auxiliar na utilização de outras habilidades numéricas que proporcionam estimativas mais precisas.

\section{Comparação entre as variáveis Aglomerado (A)/Espaçado (E) nos diferentes Anos e Escalas}

Fixando a Escala 10 e realizando a interação entre $A / E$, obteve-se que a interação entre $A / E$ e ano não é significativa para a escola pública ( $p=0,518$, média $A=0,0618$; média $E=0,0761$ ), mas é significativa para a particular ( $p=0,015$, média $A=0,0428$; média $E=0,0340$ ). Em $E 20$, a interação $A / E$ e ano não é significativa nem para a escola pública ( $p=0,318$, média $A=0,0618$; média $E=0,0761$ ), nem para a particular ( $p=0,064$; media $A=0,0380$; média $E=0,0560$. Analisada a E100, obteve-se a interação A/E e ano significativa $(p=0,017)$ para a escola pública (média $A=0,0731$; média $E=0,1072$ ), mas não significativa para a particular $(p=0,487$; média $A=0,0438$; média $E=0,0799)$. Desconsidera-se a interação significativa obtida na escala 10, levando em conta que itens aglomerados ou espaçados em uma escala pequena podem não fazer sentido. As médias 
apresentadas nos diferentes anos para itens aglomerados ou espaçados, na escala 100, são apresentadas na Tabela 6.

Tabela 6. Médias de PR para itens Aglomerados (A)/Espaçados (E) por ano escolar e escola em E100

\begin{tabular}{cccccc}
\hline \multirow{2}{*}{ Ano } & \multicolumn{2}{c}{ Escola Pública } & & \multicolumn{2}{c}{ Escola Privada } \\
\cline { 2 - 3 } \cline { 6 - 6 } & $\mathrm{A}$ & $\mathrm{E}$ & & $\mathrm{A}$ & $\mathrm{E}$ \\
\hline $2^{\circ}$ & 0,1319 & 0,1514 & & 0,0626 & 0,0913 \\
$3^{\circ}$ & 0,1049 & 0,1304 & & 0,051 & 0,0866 \\
$4^{\circ}$ & 0,0974 & 0,1328 & & 0,0466 & 0,0795 \\
$5^{\circ}$ & 0,0671 & 0,1023 & & 0,0406 & 0,0779 \\
$6^{\circ}$ & 0,0474 & 0,0936 & & 0,0429 & 0,0838 \\
\hline
\end{tabular}

Na escala 100, na escola pública, obteve-se um grupo de estudantes do $2^{\circ}$ ao $4^{\circ}$ ano com médias estatisticamente nāo diferentes para itens aglomerados e espaçados. $05^{\circ}$ ano diferiu sua precisão do $6^{\circ}$ ano para itens aglomerados, mas nāo para espaçados. Corroborando as conclusōes apresentadas para habilidades matemáticas serem utilizadas para a matriz conhecida, itens apresentados de maneira aglomerada também podem proporcionar que essas habilidades sejam acionadas, buscando no raciocínio aditivo e multiplicativo subsídios para melhorar a precisão das estimativas. Os grupos formados por nível de precisão de estimativas, para esses estudantes, são descritos na Tabela 7.

Tabela 7. Categorizaçāo dos anos escolares por Níveis de média de Precisāo Relativa para itens Aglomerados (A) e Espaçados (E) na escola pública e na Escala 100

\begin{tabular}{|c|c|c|c|c|c|c|c|}
\hline \multirow{3}{*}{ Escala } & \multirow{2}{*}{ Nível } & \multicolumn{6}{|c|}{ Escola Pública } \\
\hline & & \multicolumn{3}{|c|}{ Aglomerados } & \multicolumn{3}{|c|}{ Espaçados } \\
\hline & N1 & $2^{\circ}$ & $3^{\circ}$ & $4^{\circ}$ & $2^{\circ}$ & $3^{\circ}$ & $4^{\circ}$ \\
\hline \multirow{2}{*}{$\begin{array}{c}\text { Escala } \\
100\end{array}$} & N2 & $5^{\circ}$ & & & $5^{\circ}$ & $6^{\circ}$ & \\
\hline & N3 & $6^{\circ}$ & & & & & \\
\hline
\end{tabular}


Nesta categorização é possível perceber semelhanças entre os grupos formados para as diferentes densidades, exceto pela superioridade da precisão apresentada pelos alunos do $6^{\circ}$ ano, no caso dos itens aglomerados. Entretanto, essas conclusōes não puderam ser confirmadas quando comparados os alunos da escola privada.

\section{Comparação entre as variáveis Aglomerado (A)/Espaçado (E) e Máximo Conhecido (MC)/Máximo Desconhecido (MD) nos diferentes Anos e Escalas}

Fixando as escalas 10 e 20 e realizando a interação entre ano, A/E, MD/MC, obteve-se que essa interação não é significativa em nenhuma das escolas: nem na pública ( $p=0,369 ; p=0,932)$, nem na escola particular $(p=0,347 ; p=0,669)$. Quando analisada a escala 100, obteve-se a interação tripla MD/MC, A/E e ano significativa em ambas as escolas, com $p<0,001$. As médias apresentadas nos diferentes anos e escalas, quando o máximo é conhecido ou não, nas distribuiçōes aglomerada ou espaçada, são apresentadas na Tabela 8.

Tabela 8. Média de PR na interaçāo entre as variáveis: Ano, A/E e MC/MD

\begin{tabular}{|c|c|c|c|c|c|c|c|c|c|}
\hline \multirow{3}{*}{ Ano } & \multirow{3}{*}{ Escala } & \multicolumn{4}{|c|}{ Máximo Desconhecido } & \multicolumn{4}{|c|}{ Máximo Conhecido } \\
\hline & & \multicolumn{2}{|c|}{ Pública } & \multicolumn{2}{|c|}{ Privada } & \multicolumn{2}{|c|}{ Pública } & \multicolumn{2}{|c|}{ Privada } \\
\hline & & E & A & E & A & E & A & E & $A$ \\
\hline \multirow[t]{3}{*}{$2^{\circ}$} & E10 & ,0693 & ,0898 & ,0598 &, 0504 &, 0864 & ,1014 &, 0662 &, 0583 \\
\hline & E20 & ,1188 & ,1157 &, 0716 & ,0691 & ,1275 & ,1341 &, 0660 & ,0443 \\
\hline & E100 & ,1602 & ,1327 & ,1079 & ,0795 &, 1426 & ,1312 &, 0748 &, 0458 \\
\hline \multirow[t]{3}{*}{$3^{\circ}$} & E10 &, 0587 &, 0870 &, 0406 & 0510 & ,1054 & ,1303 &, 0604 &, 0442 \\
\hline & E20 & ,1051 & ,0916 &, 0632 &, 0458 &, 1025 &, 0809 &, 0516 &, 0247 \\
\hline & E100 & ,1361 &, 0984 & 1057 &, 0648 &, 1246 & ,1115 &, 0675 &, 0371 \\
\hline \multirow[t]{3}{*}{$4^{\circ}$} & E10 &, 0522 &, 0690 &, 0356 &, 0500 &, 1057 & ,0922 &, 0295 &, 0637 \\
\hline & E20 &, 0874 & 0757 &, 0635 & 0580 &, 0904 &, 0860 & ,0425 & ,0287 \\
\hline & E100 & 1507 & 1309 & 1111 &, 0666 & 1143 & ,0621 & ,0480 & ,0268 \\
\hline
\end{tabular}




\begin{tabular}{cccccccccc}
\hline $5^{\circ}$ & E10 &, 0422 &, 0481 &, 0335 &, 0476 &, 0368 &, 0410 &, 0285 &, 0283 \\
& E20 &, 0642 &, 0553 &, 0559 &, 0383 &, 0499 &, 0340 &, 0455 &, 0248 \\
& E100 &, 1395 &, 0938 &, 1050 &, 0581 &, 0640 &, 0399 &, 0512 &, 0230 \\
\hline $6^{\circ}$ & $E 10$ &, 0339 &, 0470 &, 0294 &, 0407 &, 0235 &, 0250 &, 0204 &, 0312 \\
& $E 20$ &, 0445 &, 0389 &, 0508 &, 0477 &, 0326 &, 0232 &, 0286 &, 0212 \\
& E100 &, 1210 &, 0582 &, 1213 &, 0607 &, 0658 &, 0365 &, 0467 &, 0252 \\
\hline
\end{tabular}

De modo geral, as médias de PR dos alunos da escola privada são menores que a dos estudantes da escola pública, o que significa que os alunos são mais precisos. Mas, em ambas as escolas, a precisão tende a ser melhor nos estudantes mais velhos, independentemente das condiçōes apresentadas. As diferenças significativas encontradas entre a precisão apresentada pelos estudantes da escola pública e da escola particular, obtidas na interação tripla para escala 100, estão destacadas na Tabela 9.

Tabela 9. Diferenças significativas entre as médias de Precisāo Relativa nos anos escolares, fixando: Aglomerados (A) / Espaçados (E), Máximo Conhecido (MC) / Máximo Desconhecido (MD) e Escola

\begin{tabular}{|c|c|c|c|c|c|}
\hline $\mathrm{A} / \mathrm{E}$ & $\mathrm{MC} / \mathrm{MD}$ & \multicolumn{2}{|c|}{ ANO } & Pública (p) & Privada (p) \\
\hline \multirow{11}{*}{ A } & \multirow{6}{*}{ MD } & \multirow{2}{*}{$2^{\circ}$} & $5^{\circ}$ & 0,006 & \\
\hline & & & $6^{\circ}$ & $<0,001$ & \\
\hline & & $3^{\circ}$ & $6^{\circ}$ & 0,003 & \\
\hline & & \multirow{2}{*}{$4^{\circ}$} & $5^{\circ}$ & 0,022 & \\
\hline & & & $6^{\circ}$ & $<0,001$ & \\
\hline & & $5^{\circ}$ & $6^{\circ}$ & $<0,001$ & \\
\hline & \multirow{5}{*}{ MC } & \multirow{3}{*}{$2^{\circ}$} & $4^{\circ}$ & 0,002 & \\
\hline & & & $5^{\circ}$ & $<0,001$ & 0,001 \\
\hline & & & $6^{\circ}$ & $<0,001$ & \\
\hline & & \multirow{2}{*}{$3^{\circ}$} & $5^{\circ}$ & $<0,001$ & \\
\hline & & & $6^{\circ}$ & $<0,001$ & \\
\hline
\end{tabular}




\begin{tabular}{|c|c|c|c|c|c|}
\hline \multirow{8}{*}{ E } & $M D$ & $2^{\circ}$ & $6^{\circ}$ & 0,002 & \\
\hline & \multirow{7}{*}{$M C$} & \multirow{3}{*}{$2^{\circ}$} & $4^{\circ}$ & & 0,006 \\
\hline & & & $5^{\circ}$ & $<0,001$ & 0,011 \\
\hline & & & $6^{\circ}$ & $<0,001$ & 0,001 \\
\hline & & \multirow{2}{*}{$3^{\circ}$} & $5^{\circ}$ & 0,002 & \\
\hline & & & $6^{\circ}$ & 0,008 & 0,043 \\
\hline & & \multirow{2}{*}{$4^{\circ}$} & $5^{\circ}$ & 0,011 & \\
\hline & & & $6^{\circ}$ & 0,039 & \\
\hline
\end{tabular}

p - Nível de confiança

Finalmente, a interação tripla traz informaçōes complementares sobre as situaçōes particulares entre as combinaçōes dos problemas apresentados. Fazendo uma análise do tipo específico de problema, por ano escolar, em cada escola (Tabela 9), obtém-se um padrão geral de desempenho em estimativa de quantidades. A tabela 10 indica os grupos categorizados.

Tabela 10. Categorizaçāo dos anos escolares por Níveis de PR, fixando-se: MD/MC, A/E e Escola

\begin{tabular}{|c|c|c|c|c|c|c|c|c|c|c|c|c|c|}
\hline Escola & $\mathrm{MC} / \mathrm{MD}$ & $\mathrm{A} / \mathrm{E}$ & & & N1 & & & & $\mathrm{N} 2$ & & & N3 & N4 \\
\hline \multirow{4}{*}{$\mathrm{Pu}$} & \multirow{2}{*}{ MD } & A & 2 & & $4^{\circ}$ & & & $3^{\circ}$ & $5^{\circ}$ & & $6^{\circ}$ & & \\
\hline & & $E$ & 2 & $3^{\circ}$ & $4^{\circ}$ & $5^{\circ}$ & & $3^{\circ}$ & $4^{\circ}$ & $5^{\circ} \quad 6^{\circ}$ & $6^{\circ}$ & & \\
\hline & \multirow{2}{*}{$M C$} & $A$ & 2 & $3^{\circ}$ & & & & $3^{\circ}$ & $4^{0}$ & & $4^{\circ}$ & $5^{\circ} \quad 6^{\circ}$ & \\
\hline & & E & 2 & $3^{\circ}$ & & & & $3^{\circ}$ & $4^{\circ}$ & & $5^{\circ}$ & $6^{\circ}$ & $6^{\circ}$ \\
\hline \multirow{4}{*}{$\mathrm{Pa}$} & \multirow{2}{*}{ MD } & $A$ & & $3^{\circ}$ & $4^{\circ}$ & $5^{\circ}$ & $6^{\circ}$ & & & & & & \\
\hline & & E & 2 & $3^{\circ}$ & $4^{\circ}$ & $5^{\circ}$ & $6^{\circ}$ & & & & & & \\
\hline & \multirow{2}{*}{ MC } & A & & $3^{\circ}$ & $4^{\circ}$ & $6^{\circ}$ & & $3^{\circ}$ & $4^{0}$ & $5^{\circ} \quad 6^{\circ}$ & & & \\
\hline & & $E$ & 2 & $3^{\circ}$ & $4^{\circ}$ & & & $3^{\circ}$ & $4^{\circ}$ & $5^{\circ}$ & $4^{\circ}$ & $5^{\circ} \quad 6^{\circ}$ & \\
\hline
\end{tabular}

Com os resultados até então encontrados, considera-se importante realizar uma comparação quádrupla entre as variáveis $\mathrm{A} / \mathrm{E}, \mathrm{MC} / \mathrm{MD}$ por ano e por escala. Neste caso, somente na escala 100 essa interação foi significativa. 


\section{DISCUSSĀO}

Como resultado geral, observou-se que há um aumento qualitativo em termos de precisão em estimativa numérica, conforme avançam os anos escolares, tal como o previsto, já que a discriminação entre magnitudes numéricas não simbólicas continua a se tornar mais precisa, bem além da infância (Halberda e Feigenson 2008). Entretanto, os diferentes níveis destacados nas diferentes situaçōes apresentadas em cada tarefa do teste apresentaram padrōes distintos, em especial, nas duas escolas. Neste caso, mais grupos foram gerados pelos estudantes da escola pública, sendo que grupos intermediários constantemente faziam parte de grupos de transição que poderiam pertencer a mais de um grupo ao mesmo tempo, indicando que, naquele ano escolar, os alunos ainda estivessem em uma etapa de transição de nível.

Esses resultados vão ao encontro da ideia de que crianças aprendem sobre as magnitudes dos números simbólicos surpreendentemente lentamente, já que sabem contar muito antes de entender as magnitudes relativas dos números que estão contando (Siegler, e Braithwaite, 2016). Os grupos de transiçāo, mais presentes nos estudantes de escola pública, também dão indícios dessa compreensão mais lenta das magnitudes. $\mathrm{E}$, se, de fato o conhecimento da magnitude numérica estiver causalmente relacionado à aritmética (Siegler, e Braithwaite, 2016), pode-se considerar que intervençōes que estimulem a compreensão dessas magnitudes numéricas também devem melhorar a aprendizagem aritmética.

Observou-se uma diferença estatisticamente consistente entre os resultados obtidos na precisão relativa dos estudantes das diferentes escolas: pública e privada. Sendo que os estudantes da escola privada tiveram estimativas mais precisas que os estudantes da escola pública, em todas as situaçōes testadas. Este fato revela uma importante constatação de que as diferenças culturais representadas pelas diferentes escolas podem apresentar mais uma evidência de que a habilidade em estimativa numérica depende do contexto e que pode ser influenciada por fatores externos, não sendo considerada uma habilidade unicamente inata. Esta constatação vem ao encontro dos resultados obtidos em estudos realizados em diferentes culturas, que evidenciaram que fatores culturais e linguísticos podem permitir que as crianças sejam mais ou menos hábeis em realizar estimativas, tais como os estudos de Cirino e Colaboradores (2016), Kersey e colaboradores (2018), Laski e Yu, 2014 e Siegler e Braithwaite (2016), contradizendo a hipótese 
levantada por Siegler e Opfer (2003), de que a estimativa numérica é uma medida pura e que independe de conhecimentos externos.

Embora os alunos do $2^{\circ}$ e $3^{\circ}$ ano conheçam quantidades maiores que 100 e possam gerar estimativas corretamente para o número pontos nesta escala, muitos demonstraram dificuldade em realizar padrōes comparativos de quantidades até 100, ou a falta de estratégias de realizar estimativas, em especial, para os itens apresentados de forma aleatória. Ou seja, os dados fornecem evidências de que alguns estudantes não compreendem a dimensão relativa de números maiores que 100, e poucos estudos sobre estimativa de quantidades ou mesmo de numerosidade foram tão longe em termos quantitativos (Kim, Jang e Cho, 2018). De acordo com os achados de Obersteiner e colaboradores (2014) sugere-se que as crianças de $2^{\circ}$ ao $6^{\circ}$ ano são mais precisas quando os pontos são apresentados em matriz do que quando eles são apresentados em arranjos aleatórios. A partir de dados sobre conhecer o máximo da matriz, verificou-se que este é um fator que contribui para diferenças no agrupamento dos estudantes da escola particular, gerando, inclusive, um nível a mais de categorização.

Uma previsão da teoria integrada proposta por Siegler e Braithwaite (2016) é que o conhecimento da magnitude numérica deve ser correlacionado com a proficiência aritmética e preditiva dela. Então, o fato de as crianças conhecerem e até operarem com números dessa magnitude, as crianças podem memorizar fatos e procedimentos aritméticos sem entender o que essas magnitudes representam. De fato, muitos educadores de matemática lamentam que a memorização mecânica de procedimentos aritméticos seja exatamente o que a maioria dos estudantes faz e essa generalização excessiva dos procedimentos aritméticos faz com o que o sujeito não reflita sobre a magnitude dos números (Siegler, e Braithwaite, 2016). Supōe-se, dessa forma, que avaliar como as crianças estimam quantidades não só pode gerar um preditivo para sua competência matemática, mas também pode dar indícios de um aprendizado mecânico ou incompleto da magnitude numérica, proporcionando a reflexão docente.

Na escala 10 e na escala 20, como esperado, os alunos tendem a realizar estimativas mais precisas, gradualmente, em cada ano escolar, em qualquer uma das situaçōes apresentadas. Entretanto, a precisāo só é realmente melhorada no $5^{\circ}$ e no $6^{\circ}$ ano, comparados aos alunos menores, sendo que estes alunos tendem a utilizar melhor a informação sobre a quantidade máxima de pontos. Considera-se que as respostas mais relevantes sejam buscadas dentro da escala 100, tendo em vista que ela possui uma variedade maior de tarefas e de números a serem estimados, e, mesmo assim, encontra-se em uma faixa numérica com a 
qual a grande maioria dos sujeitos pesquisados está familiarizada. Então, conclui-se que conhecer o máximo de pontos da matriz é uma informaçāo importante para realizar estimativas em grandes escalas, o que leva a supor que relaçōes matemáticas que exigem conhecer o máximo de pontos são utilizadas em todos os níveis escolares.

De fato, os estudantes mais velhos podem utilizar-se de habilidades aritméticas de adição e subtração e mesmo de raciocínio proporcional com a informação do limite da matriz para estimar a quantidade de pontos nela distribuídos. As relaçōes entre quantidades estabelecidas por um raciocínio aditivo (parte-todo) e multiplicativo (correspondência um para muitos), no sentido proposto por Nunes e colaboradores (2016) e Nogues e Dorneles (2020), já construídos possibilitam a elaboração inconsciente de estratégias de estimativa mais complexas, eficientes e precisas.

Realizando a interação entre as variáveis de formato de apresentação, aglomerado ou espaçado, com o ano escolar, obteve-se resultado positivo apenas na escala 100 e para os estudantes de escola pública. Em especial, para escalas pequenas, faz pouco sentido falar em itens aglomerados e espaçados, tendo em vista o tamanho da matriz e as quantidades apresentadas. Sendo assim, considera-se que esses resultados não sejam úteis na compreensão do desempenho das precisōes com estas variáveis. Na escola pública, a escala 100 gerou níveis de precisão que distinguiram o $6^{\circ}$ ano como os mais precisos para itens aglomerados. Na comparação quádrupla entre as variáveis de densidade (A/E) e de conhecimento do máximo da matriz (MC/MD) por ano e por escala, somente na escala 100 essa interação foi significativa, em ambas as escolas.

Esses resultados também dão suporte à ideia de que o pensamento aritmético e proporcional pode estar envolvido na habilidade de realizar estimativas e isso também poderia explicar as diferenças de precisão obtidas entre as escolas. De maneira mais geral, não está claro como a estimativa de quantidades discretas poderia contribuir para criar uma compreensão simbólica do número e suas relaçōes, já que ninguém pode distinguir consistentemente 158 de 159 pontos, mas todos que entendem o sistema decimal sabem com absoluta certeza que "159" é maior que "158" (Siegler, e Braithwaite, 2016). Entretanto, a análise da literatura sobre o tema e os resultados obtidos neste estudo indicam que o conhecimento aritmético e a estimativa de quantidades estão de alguma forma relacionados. 


\section{CONSIDERAÇÕES FINAIS}

A partir de um levantamento bibliográfico sobre habilidades matemáticas preditoras do sucesso na vida adulta, a compreensão acerca das magnitudes numéricas apresentou-se como tema central das discussōes. Alguns estudos consideram que essa é uma habilidade inata, outros entendem que é desenvolvida pela experiência. De fato, a estimativa de quantidades enquanto útil para tomadas de decisão diárias sugerem uma avaliação altamente individual e dependente do contexto magnitudes numéricas, em vez de um conjunto universal e estável do "senso numérico" (Leibovich; Katzin; Harel; e Henik, 2017). Com essas duas constataçōes: importância para o sucesso e possibilidade de construção, o objetivo central desse estudo foi identificar possíveis níveis de precisão de estimativa numérica de quantidades em estudantes do $2^{\circ}$ ao $6^{\circ}$ ano escolar.

Para alcançar esse objetivo, foram propostos três questionamentos norteadores: 1) como se dá o desenvolvimento da estimativa numérica em crianças do $2^{\circ}$ ao $6^{0}$ ano escolar em diferentes situações de apresentação dos estímulos?; 2) seria este desenvolvimento progressivo e demarcado por grupos de estudantes de mesmos anos escolares? e 3) de que maneira o modo de apresentação dos estímulos pode influenciar na precisão das estimativas nos diferentes anos escolares?

Para responder ao primeiro questionamento, a hipótese principal era de que seriam ampliados os resultados já conhecidos a respeito das crianças mais velhas realizarem estimativas com maior precisão quando comparadas às mais novas, em qualquer uma das diferentes situaçōes apresentadas. Ou seja, a habilidade de realizar estimativas seria ampliada com o passar da idade e da experiência. Essa hipótese foi confirmada, já que, para todos os diferentes modos de apresentação dos estímulos, os sujeitos mais velhos foram mais precisos que os mais novos, sendo esse desenvolvimento demonstrado por níveis de faixa etária semelhantes que foram categorizados em diferentes níveis.

Para o segundo questionamento, a hipótese de que o desenvolvimento da estimativa numérica de quantidades é progressivo e que envolve níveis de desenvolvimento semelhantes para a mesma faixa etária ou ano escolar, foi confirmada, já que foi possível estabelecer grupos de anos escolares que apresentaram precisão semelhante entre si e grupos de precisão que diferem destes e diferem por escola. Também foram observados alguns grupos de transição, pertencentes a mais de um grupo ao mesmo tempo. Esses resultados indicaram que a estimativa numérica tem seu desenvolvimento progressivo, mas diferente para cada situação e escola. Além disso, os níveis são distintos de acordo com 
as condiçōes apresentadas e com as características do grupo. Por exemplo, para uma escala menor, as crianças tornaram-se mais precisas mais cedo do que em escalas maiores.

A diferença entre a precisão obtida pelos alunos da escola pública e privada nas diferentes tarefas solicitadas indica que fatores como o nível socioeconômico e as experiências culturais, por exemplo, podem interferir nesta habilidade. Portanto, não está claro se as diferenças nos desempenhos em estimativa numérica entre estudantes de escola pública e particular estão enraizadas em diferenças intrínsecas no raciocínio estimativo ou se as diferenças emergem como resultado de diferenças na exposição cultural à estimativa Kersey, Braham, Csumitta, Libertus e Cantlon (2018) ou na própria relaçāo entre quantidades (Nunes et al., 2016).

Para a terceira questão, discutiu-se a maneira com a qual o modo de apresentação das quantidades (diferentes escalas, diferentes densidades, com ou sem matriz de referência) poderia influenciar na precisão das estimativas nos diferentes anos escolares. Quanto a isso, as hipóteses do estudo consideravam apenas os níveis de dificuldade que se julgava existir em escalas maiores. Entretanto, o que se encontrou foi que todas as variáveis envolvidas mostraram exercer um papel significativo nas estimativas dos diferentes níveis escolares, em especial para escala 100 e para itens aleatórios.

A diferença de conteúdos trabalhados e conhecimentos matemáticos também podem ter influenciado todos os resultados, comparando-se as escolas. Essas são evidências complementares que auxiliam na constatação de que estimativa numérica de quantidades não é uma medida pura e pode ser influenciada por muitos fatores. Pode-se dizer, neste caso, que há diferentes níveis de desenvolvimento da habilidade de estimativa de quantidades, mas que ocorrem em anos distintos nas diferentes escolas. Os resultados sugerem que essas diferenças se relacionam mais com escolarizaçāo do que com idade.

Esta abordagem transversal permitiu determinar que a acurácia em estimativa numérica de quantidades se desenvolve nas etapas escolares testadas. Pode-se afirmar que, até onde se sabe, que antes desse, nenhum estudo aprofundou o tema do desenvolvimento de estimativa numérica de quantidades em crianças desde a fase da alfabetização matemática ( $2^{\circ}$ ano) até o nível de formalização de conceitos matemáticos ( $6^{\circ}$ ano). No entanto, é importante destacar que este tipo de delineamento transversal pode ter sido influenciado por uma tendência do grupo e/ou pela trajetória de desenvolvimento de cada criança, desconsiderando mudanças descontínuas na precisāo em estimativa 
numérica, que somente seriam reveladas em uma análise longitudinal de seu desempenho individual ao longo do tempo.

Outra limitação do estudo foi a falta de testagem dos avanços relacionados à velocidade na realização de estimativas. Pode-se postular que as crianças mais jovens devem ter uma maior fluência com quantidades não simbólicas. Em contraste, os significados dos números simbólicos precisam ser aprendidos. Por outro lado, as quantidades simbólicas e as relaçōes numéricas e operaçōes numéricas de quantidades não simbólica também sāo fatores que agilizam o processo de estimar. Sobre estas afirmaçōes, não se pode concluir. Para os alunos mais novos, também se deve considerar os erros de registro das quantidades, a partir de confusōes sobre a escrita simbólica dos números e numerais, o que pode, inclusive, ser representado pelas quantidades representadas por números maiores que o dobro da matriz (que não foram consideradas neste estudo).

Esse, até onde se sabe, foi um primeiro estudo brasileiro a lançar um olhar para a representação não simbólica das magnitudes numéricas, no intuito de compreender (a partir da ideia de que estejam, de alguma forma, relacionadas ao conhecimento matemático posterior) se e como são construídos pelo sujeito. Acredita-se que os resultados desse estudo podem trazer implicaçōes importantes para a maneira como a matemática básica é vista e ensinada na escola, bem como para a maneira como as dificuldades matemáticas são diagnosticadas e melhoradas.

Consistente com essa visão, esse seria um primeiro passo para a compreensão dos professores sobre a relevância da estimativa numérica no contexto escolar e sobre as etapas de construção dessa habilidade, gerando conhecimento de como ensiná-la de forma eficaz. Ainda, possibilita que os professores identifiquem e freiem a ênfase muitas vezes dada à memorização mecânica de procedimentos aritméticos que dão a falsa impressāo de que os estudantes conhecem as magnitudes numéricas.

Em suma, os resultados aqui são consistentes com os trabalhos anteriores, mostrando que o desempenho em estimativa numérica de quantidades está relacionado a uma grande variedade de preditores no domínio cognitivo. Esse estudo contribui para ampliar o conhecimento sobre o desenvolvimento numérico em crianças. Esses ganhos incluem maior compreensão do desenvolvimento da capacidade de realizar estimativa de quantidades discretas e dá indícios de que está, de alguma forma, relacionado às relaçōes numéricas aritméticas. 


\section{REFERÊNCIAS}

Brysbaert, M. (2005). Number Recognition in Different Formats. In Campbell, J. I. D. Handbook of mathematical cognition. (Chap. 2, pp. 23-42). Psychology Press.

Cirino, P. T., Tolar, T. D., Fuchs, L. S., e Huston-Warren, E. (2016). Cognitive and numerosity predictors of mathematical skills in middle school. Journal of Experimental Child Psychology. 145, 95-119.

Corso, L., e Dorneles, B. V. (2012). Qual o Papel que a Memória de Trabalho Exerce na Aprendizagem da Matemática? Bolema. Boletim de Educaçāo Matemática. 26(42b), 627-647.

Dehaene, S. (1997). The Number Sense: how the mind creates mathematics. Oxford University Press.

Dorneles, B. V., Duro, M. L., dos Santos, S. N., Pisacco, N. M. T., Sperafico, Y. L. S., e Enricone. J. R. B. (2015, agosto). Number Estimation in Children Assessed with a No-number-line Estimation Task. Anais do Biennial EARLI Conference, Limassol, Cyprus, 16.

Feigenson, L., Dehaene, S. e Spelke, E. (2004) Core systems of number. Trends in Cognitive Sciences 8(7):307-14.

Gebuis, T., e Reynvoet, B. (2012). The Role of Visual Information in Numerosity Estimation. PLoSOne, 7(5).

Halberda, J., e Feigenson, L. (2008). Developmental Change in the Acuity of the "Number Sense": the approximate number system in 3, 4, 5, and 6-year-olds and adults. Developmental Psychology. 44, 1457-1465.

Hurley, E., Boykin, W., e Allen, B. (2005). Communal Versus Individual Learning of a Math-Estimation Task: African American Children and the Culture of Learning Contexts. The Journal of Psychology, 139(6), 513-527.

Kersey, A. J, Braham, E. J., Csumitta, K. D., Libertus, M. E, e Cantlon, J. F. (2018). No intrinsic gender differences in children's earliest numerical abilities. Science of Learning. 3(12).

Kim, N., Jang, S., e Cho, S. (2018) Testing the Efficacy of Training Basic Numerical Cognition and Transfer Effects to Improvement in Children's Math Ability Frontiers in Psychology. 9, 1775.

Laski, E., e Yu, Q. (2014). Number Line Estimation and Mental Addition: examining the potential roles of language and education. Journal of Experimental Child Psychology, 117, 29-44.

Leibovich, T., Katzin, N., Harel, M., e Henik, A. (2017). From "sense of number" to "sense of magnitude": The role of continuous magnitudes in numerical cognition. Behavioral and Brain Sciences, 1-62. 
Nogues, C. P., e Dorneles, B. V. (2020). Estimativa numérica, memória de trabalho e raciocínio quantitativo: relaçōes no desempenho matemático. Zetetiké, Campinas: SP, 1-17

Nunes, T., Dorneles, B. V., Lin, P.-J., e Rathgeb-Schnierer, E. (2016). Teaching and learning about whole numbers in Primary School. In ICME-13 Topical Surveys (Springer O).

Obersteiner, A., Reiss, K., Ufer, S., Luwel, K., e Verschaffel, L. (2014). Do First Graders Make Efficient Use of External Number Representations? The Case of the Twenty-Frame. Cognition and Instruction, 32(4), 353-373.

Opfer, J., e Siegler, R. (2012). Development of Quantitative Thinking. In Holyoak, K. e Morrison R. (Eds.), Oxford Handbook of Thinking and Reasoning. Oxford University Press.

Piazza, M., Mechelli, A., Price, C. J., e Butterworth, B. (2006). Exact and Approximate Judgments of Visual and Auditory Numerosity: An fMRI study. Brain Research. 1106, 177-188.

Plicastro, M. S., Rodrigues, A. A., e Ribeiro, M. (2017). Conhecimento especializado revelado por professores da educação infantil dos anos iniciais no tema de medida de comprimento e sua estimativa. Espaço Plural. 6, 123-154.

Rousselle, L., e Noel, M. P. (2008). The Development of Automatic Numerosity Processing in Preschoolers: evidence for numerosity-perceptual interference. Developmental Psychology. 44(2), 544-560.

Schneider, M., Grabner, R., e Paetsch, J. (2009). Mental Number Line, Number Line Estimation, and Mathematical Achievement: their interrelations in grades 5 and 6. Journal of Educational Psychology. 101, 359-372.

Siegler, R., e Booth, J. (2004). Development of Numerical Estimation in Young Children. Child Development. 75, 428-444.

Siegler, R. S., e Braithwaite, D. W. (in press). Numerical development. Annual Review of Psychology. (Anticipated publication: 2016.)

Siegler, R., e Opfer, J. (2003). The Development of Numerical Estimation: evidence for multiple representations of numerical quantity. Psychological Science. 14, 237-243.

Silva, M. D. B., Farias, S. G., e Lima, E. M. (2020) Estimativa Numérica: Estado da arte. Revista Científica Multidisciplinar Núcleo do Conhecimento. 01(3), 107-120.

MARIANA LIMA DURO

Dirección: Instituto Federal do Rio Grande do Sul (IFRS) - Campus Canoas Rua Maria Zélia Carneiro de Figueiredo, 870-A, Bairro Igara III Canoas/RS - CEP"92412-240

Teléfono: +55513415-8200 ou +55 51999896769 